\title{
ACTIVE STEERING OF RAILWAY VEHICLES VIA DOBC TECHNIQUES
}

\section{Dong HU*, Wen-Hua CHEN* and Roger M. GOODALL**}

\author{
*Department of Aeronautical and Automotive Engineering, \\ Loughborough University, Leicestershire, UK, LE11 $3 T U$. \\ Email:d.hu@lboro.ac.uk,w.chen@lboro.ac.uk \\ **Department of Electronic and Electrical Engineering, \\ Loughborough University, Leicestershire, UK, LE11 3TU, \\ Email:R.M.Goodall@lboro.ac.uk
}

\begin{abstract}
A novel control scheme for the active steering of railway vehicles is presented in the paper using disturbance observer based control (DOBC) techniques. In contrast to existing DOBC schemes which are mainly for singleinput-single-output (SISO) systems and designed by trial and error, a new DOBC technique for multi-input-multi-output (MIMO) systems is developed. This is achieved by reforming the DOBC into the parameterisation of all stabilising controllers and then the disturbance observer is designed to minimise the influence of disturbances. In this scheme, the disturbance observer does not have influence on tracking/regulation performance and stability. The developed DOBC scheme is then applied to the control of railway vehicles, where an LQG controller is designed as the primal controller for the vehicle running on straight tracks and the disturbance observer is employed to improve the curving performance. This overcomes the difficulty in compromising the performance and wheelset stability on straight tracks and the curving performance when a single controller is used. The simulation reveals that the curving performance of railway vehicles is improved while the straight track performance/wheelset stability remains the same. Copyright (C) 2005 IFAC
\end{abstract}

Keywords: disturbance observer, LQG control, $H_{\infty}$ control, railway vehicles

\section{INTRODUCTION}

Almost all railway vehicles use the conventional solid axle wheelset, where two coned/profiled wheels are rigidly connected to a common axle so that they have to rotate at the same speed. The advantage of this arrangement is that the wheelset has the ability of natural curving/centring, but an unconstrained solid axle wheelset is unstable and exhibits a sustained oscillation in the horizontal plane, a phenomenon known as "wheelset hunting' (Wickens (2003)). The development history of railway vehicles has evidently shown that it is dif- ficult to solve all the design difficulties effectively with only passive means. Typically a railway vehicle is stable at low speeds, but as the speed increases, a point is reached at which the vehicle becomes unstable. The coning of the wheelsets, which provides guidance, is also the source of this dynamic instability. Furthermore, variations in the coning angle of the wheelsets and the full range of vehicle operating speeds have to be considered. This is overcome on conventional railway vehicles using springs connected from wheelset to the bogie or the body of the vehicle. However, this added stiffness degrades the ability of the wheelset 
to curve and results in wear of the wheelsets and rails. It is difficult for design engineers to compromise the curving, the dynamic performance and the wheelset stability. The design aim for railway vehicles is to avoid flange contact in normal running because this is what causes high wear on the wheel and rail, which is difficult for a conventional passive vehicle design. A compromise between the wheelset stability and the curving performance has always to be made in the design of railway vehicles. Therefore, the most promising way (and arguably the only feasible way) is to take a radical approach by applying advanced control concepts via mechatronic components (Mei and Goodall (2003) references therein).

This paper advocates Disturbance Observer Based Control (DOBC) techniques for active steering of railway vehicles. As a method developed from engineering, DOBC has been successfully applied in mechatronics systems, in particular in Japan, and can deal with deterministic disturbances by means of estimating these deterministic disturbances and then feeding back them to control input of systems. The $Q$ filter in DOBC is critical for the performance. Although there are several guidelines for designing the $Q$ filter, all of them are either system-specific or only for single-inputsingle-output (SISO) systems (for example, see Schrijver and Johannes (2002); Choi et al. (2003)), and there is no systematic method for the $Q$ filter design, especially for MIMO systems. A novel DOBC design for MIMO systems is developed in this paper and then applied to dealing with the track curves and irregularities in the active steering of railway vehicles.

The paper is structured as follows. In Section 2, the linearised model of a solid 2-axle railway vehicle is given. A novel DOBC design technique for MIMO systems has been developed based on coprime factorisation theory using $H_{\infty}$ control techniques in Section 3. Thereafter, the DOBC design technique is applied to the control for active steering of railway vehicles in Section 4 . Conclusions are drawn in the last section.

\section{MODELLING OF A RAILWAY VEHICLE}

The dynamics of the railway vehicle with two solid-axle wheelsets can be approximated by a linear multi-input-multi-output (MIMO) state space model (Goodall and Li (2000); Mei and Goodall (1999), Mei and Goodall (2000b), Mei and Goodall $(2000 a))$, given by

$$
\begin{aligned}
& \dot{x}=A x+B u+T w \\
& y=C x
\end{aligned}
$$

where $x \in R^{12}, u \in R^{2}$, and $w \in R^{4}$ are the state variables, torques input and track curving input (curvature and cant angle) respectively. $T$ is a $12 \times 4$ matrix. A more detailed model can be found in the paper (Mei and Goodall (1999)). When any control strategy is adopted for the active steering of railway vehicles, the controller must stabilise the vehicle system and the closed-loop system should be robust against variation of operational parameters. Meanwhile, the controller should not interfere with the natural curving action of the solid axle wheelsets.

This is a linearised model and the real railway vehicle has profound non-linearities associated with the wheel flanges, which contact the rail when the lateral movement available on the coned section is exceeded. When an active controller is well designed, the lateral displacements would be small enough to help vehicles working in their linear regime (Goodall and $\mathrm{Li}(2000)$ ). In that case, the full nonlinear vehicle dynamics can be reasonably approximated by the linearised model (Mei and Goodall (1999), Mei and Goodall (2000a), Mei and Goodall (2003)).

Normally, the wheelsets are a instable system. Our aims are to keep the system stable and have a good curving performance. On straight rails, it is easy to keep the system stable. However, it is difficult to keep a good curving performance using a controller because of lateral displacements and lateral creep forces in which reducing lateral creep forces is the most important index. Although a number of controller may be developed, this paper proposed a new control strategy called DOBC. The obvious advantage is that the disturbance observer filter will not work on straight rails so that it does not interfere with the stability and therefore is developed independently with the controller on straight rails.

\section{DOBC FOR MIMO SYSTEMS}

The traditional DOBC structure is shown in Fig. 1 (Umeno and Hori (1991); Oh et al. (1999)), where $G$ denotes the model of the plant $P, K_{0}$ the compensator (i.e., the primal controller) and the filter $Q(s)$. The disturbance observer deduces disturbances from the difference between the reconstructed plant input and the plant input coming from the compensator $K_{0}$. In almost all applications of DOBC techniques, the plants are minimum phase and SISO systems. The estimated disturbance $\hat{d}_{0}$ is added to the output of the compensator to attenuate the disturbance $d_{i}$. The filter $Q(s)$ plays an important role in DOBC since it not only makes the disturbance observer stable and realisable but also has significant influence on DOBC performance. Therefore, how to design $Q(s)$ filters is one of the main focuses in DOBC study. The general guideline is that $Q(s)$ is a 


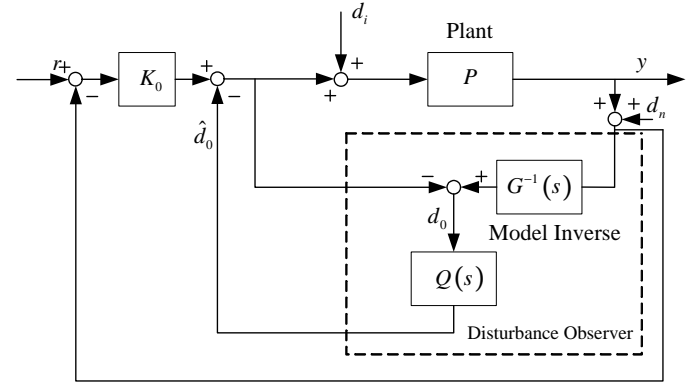

Fig. 1. The traditional disturbance observer based control structure with a compensator

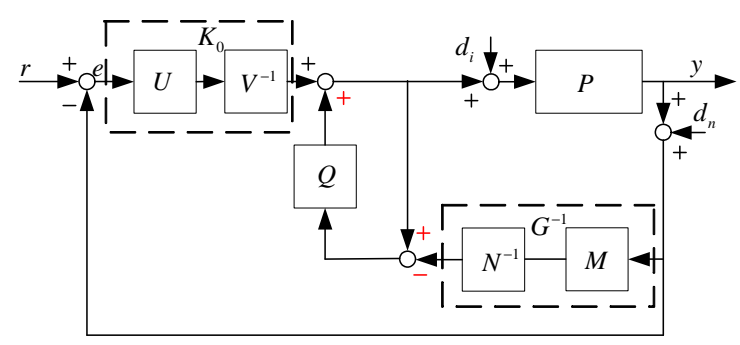

Fig. 2. DOBC decomposition

low pass filter with appropriate cut-off frequency (Umeno and Hori (1991)). For a specific class of SISO systems, some more detailed guidelines have been presented, for example for second order systems in Choi et al. (2003). Up to now, there are no systematic methods for $Q(s)$ design, in particular for MIMO systems like railway vehicles considered in this paper. A new method to design $Q(s)$ will be presented in this section.

\subsection{Equivalent structure of DOBC}

With reference to Fig. 1, provided that $P=G$, let $G=M^{-1} N=\tilde{N} \tilde{M}^{-1} \quad K_{0}=V^{-1} U=\tilde{U} \tilde{V}^{-1}$ where $M, N, \tilde{N}, \tilde{M}, V, U, \tilde{U}, \tilde{V} \in R H_{\infty}$ satisfy a doubly Bezout equation

$$
\left[\begin{array}{cc}
V & U \\
-N & M
\end{array}\right]\left[\begin{array}{cc}
\tilde{M} & -\tilde{U} \\
\tilde{N} & \tilde{V}
\end{array}\right]=\left[\begin{array}{ll}
I & 0 \\
0 & I
\end{array}\right]
$$

and $R H_{\infty}$ denotes the space of real rational complex-valued functions that are stable and proper. With these notations, the traditional structure of DOBC can be represented in a more general form as in Fig. 2.

Using block diagram manipulation, the diagram in Fig. 1 can be further represented as in Fig. 3 where $Q_{2}=V Q N^{-1}$. This implies that the traditional DOBC structure can be represented in the all internally stabilising controllers structure. In fact, we will prove that if the nominal controller $K_{0}$ is a stabilising controller, the closed-loop system with the structure in Fig. 3 is always stable for any stable $Q_{2}$. This parameterises all the stablising DOBC by the newly introducing filter $Q_{2}(s)$ and provides a more general approach for the design of

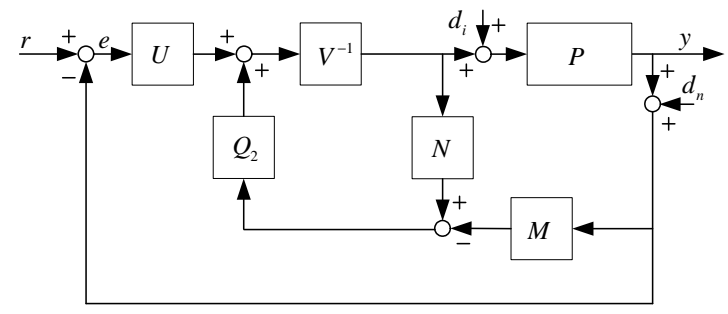

Fig. 3. DOBC new structure

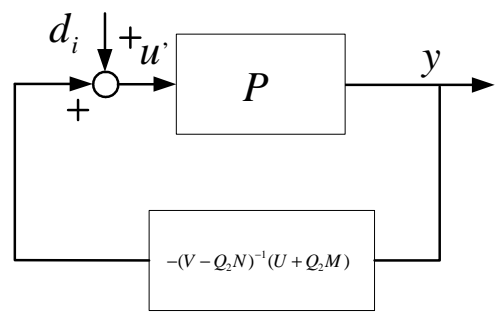

Fig. 4. Sensitivity solution to the design of disturbance observer

the filter $Q(s)$ in the traditional DOBC structure (Umeno and Hori (1991); Chen et al. (2000), therein references).

Lemma 1. (Vidyasagar (1985)) A necessary and sufficient condition to ensure internal stability of a general feedback control loop is that

$$
\left[\begin{array}{cc}
I & -K \\
-G & I
\end{array}\right]^{-1} \in R H_{\infty}
$$

we say that $K$ stabilises $G$ or $(G, K)$ is a stabilising pair if Equation (4) holds.

Theorem 2. Consider a general DOBC structure in Fig. 1, where $N, M, V$ and $U$ satisfying the doubly Bezout equation (3) are coprime factorisation of the plant model $G$ and the primal controller $K_{0}$ that stabilises the plant model $G$. The closedloop system under the DOBC is always stable for any stable proper transfer function $Q_{2}=V Q N^{-1}$ satisfying $V(\infty)-Q_{2}(\infty) N(\infty) \neq 0$, i. e. $\operatorname{det}(V-$ $\left.Q_{2} N\right) \neq 0$.

Since the controller $K_{0}$ internally stabilises the plant model $P$, it follows from Lemma 1 that $\left[\begin{array}{cc}I & K_{0} \\ -G & I\end{array}\right]^{-1} \in R H_{\infty}$ for any proper stable $Q_{2}$. Furthermore, $N, M, \tilde{N}$ and $\tilde{M}$ are proper and stable. Together with Lemma 1, this implies that the closed-loop system under the DOBC in Fig. 3 is always stable for any proper stable $Q_{2}$.

Corollary 3. Referring to the traditional DOBC structure in Fig. 1, the closed-loop system under DOBC is always stable if the controller $K_{0}$ stabilises a system $G$ and the $Q$ filter is given by $Q=V^{-1} Q_{2} N$ for any proper stable $Q_{2}$ where $V$ and $N$ are coprime fatorisation of the plant model and the compensator $K_{0}$ and $Q_{2}$ satisfies $V(\infty)-$ $Q_{2}(\infty) N(\infty) \neq 0$, i. e. $\operatorname{det}\left(V-Q_{2} N\right) \neq 0$. 
Theorem 2 and its corollary state that all the stabilising controllers in the DOBC structure can be parameterised by the new stable filter $Q_{2}(s)$. Furthermore, it can be proved that under the DOBC structure without disturbances, the transfer function from the input to the output is given by

$$
\frac{y(s)}{r(s)}=G K_{0}\left(I+G K_{0}\right)^{-1}
$$

which is independent of the filter $Q$ (and hence $Q_{2}$ ). This implies that the choice of the disturbance filter does not have influence on the tracking or regulation performance and the filter $Q$ is solely chosen for the purpose of disturbance attenuation. Hence the design of the primal controller $K_{0}$ is completely separated from the design of the disturbance observer. This is the very important feature of the DOBC structure. Selecting the filter $Q$ (and hence $Q_{2}$ ) does not change the transfer function between reference $r$ and output $y$. This feature together with the new approach to parameterising all internally stablising disturbance observer based controllers by the new filter $Q_{2}$ provides a general approach for DOBC design (see Fig. 4).

\subsection{Q filter design}

As discussed in Section 3.1, one of the main focuses in DOBC is to design the filter $Q$ (and hence $Q_{2}$ ). The theoretic result developed in the last section permits us to develop a systematic approach for MIMO systems. Since the DOBC is stable as long as $Q_{2}$ is proper stable, i.e. $Q_{2} \in R H_{\infty}$, the disturbance observer can be designed using $H_{\infty}$ theory in terms of $Q_{2}(s)$ (and hence $Q$ ). More specifically, to attenuate the influence of the disturbances, the design is performed by minimising the $H_{\infty}$ norm of the transfer function matrix from the disturbances to the outputs in terms of $Q_{2}$ and, according to Theorem 2 and Corollary 3 , the stability of the closed-loop system under the DOBC with $Q=V^{-1} Q_{2} N$ is guaranteed for any $Q_{2}$ yielded by $H_{\infty}$ optimisation (Zhou and Doyle (1998)).

For a system model $G$, a minimal realisation of $G$ is $[A, B, C, 0]$. Assume that $F, L$ are the matrices such that two systems $((A-B F),[B$ $\left.L],[F C]^{\prime}\right)$ and $\left((A-L C),[B L],[F C]^{\prime}\right)$ are inputoutput stable. Then it is easy to constitute a doubly coprime factorisation of $G$ and an LQG controller $K_{0}$ which stabilises the system (Nett et al. (1984)).

According to the new DOBC techniques in Section 3.1 , if there are no disturbances, the performance of the system is determined by the controller $K_{0}$.

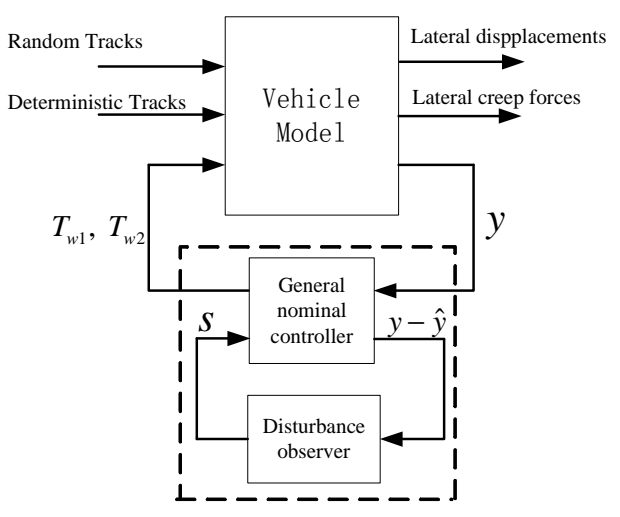

Fig. 5. Structure diagram of DOBC control for vehicles

When disturbances are present, the filter $Q_{2}$ takes action and it does not affect the tracking and regulation performance of the controller $K_{0}$. The filter $Q_{2}$ is designed to reduce the effect of disturbances $d$ on outputs by minimising sensitivity functions between disturbances and outputs. $H_{\infty}$ design techniques can be used to optimise the sensitivity between disturbances $d$ and outputs in Fig. 4 due to $Q_{2} \in R H_{\infty}$. The $Q_{2}$ design is readily performed by existing $H_{\infty}$ design tools such as Linear Matrix Inequalities (LMIs) (for example, see MathWorks (1997); Boyd et al. (1994)).

\section{ACTIVE STEERING OF RAILWAY VEHICLES VIA DOBC TECHNIQUES}

A diagram of the active railway vehicle control using the DOBC structure is shown in Fig. 5. In contrast to existing active steering strategies (Mei and Goodall (2003) references therein) where a single control is used to achieve good performance for railway vehicles running on both straight and curved tracks and it is difficult not to interfere with the natural curving action of the solid axle wheelsets, a primal controller is designed for the stability/performance for railway vehicles running on straight tracks and the disturbance observer is designed to improve the curving performance on curved tracks in the DOBC strategy. In this application, the curved tracks and other factors are considered as disturbance and the disturbance observer is designed to minimise their influence on the stability of the riding performance of railway vehicles.

\subsection{Design of LQG controller}

First the controller for the railway vehicles running on straight tracks is designed using the Linear Quadratic Gaussian (LQG) control technique as the paper (Mei and Goodall (1999)). A curved track with radius of $1000 \mathrm{~m}$ connected to straight tracks on either end via a transition is selected to 
study the control performance on curved tracks (Mei and Goodall (1999)). A time delay is set to reflect the difference between the leading and trailing wheelsets. The track is canted inwards to give a zero cant deficiency, i.e. zero lateral acceleration at the running speed of $60 \mathrm{~m} / \mathrm{s}$.

The curved performance is not satisfactory (see Mei and Goodall (1999)). Normally, the railway vehicles run on the straight rail tracks only using the simpler primal LQG controller to guaranteed the whole system stable. However, when the vehicles run on curved tracks, the dynamic performance on lateral displacements and yaw angles can not be accepted, thus the more control effort should be afforded to obtain a good curving performance. The primal LQG can work well on straight tracks. However, if the more control effort is afforded on straight tracks, some other indices will be improved. How to separate the stability and curving performance control design? DOBC techniques can provide a good solution.

\section{2 $D O B C$}

The above designed LQG controller is used as the primal controller $K_{0}$ for straight track performance. Then following the procedure in Section 3, the disturbance observer is designed to improve the curving performance of the primal LQG controller instead does not work on straight tracks (see Fig. 5). With the state space realisation of the designed controller in Section 3.2 and the vehicle model as in Section 2, the filter $Q_{2}$ (hence $Q$ ) is designed using LMI control toolbox for MATLAB. When the railway vehicle runs on the straight tracks, the measured lateral displacements $y$ are equal to the estimated lateral displacements $\hat{y}$ which is constituted from the state estimate yielded by the built-in Kalman filter in Section 4.1, no signal is input to the disturbance filter. When the track curves and irregularities are present, the filter $Q_{2}$ (hence $Q$ ) is designed by minimising the $H_{\infty}$ norm of the transfer function matrix from the track curves and irregularities to the lateral displacements and later creep forces, so that a good curving performance is achieved. This filter $Q(s)$ in DOBC implementation is then obtained. It is noticed that the performance of the active different steering strategies are evaluated using a set of criteria (Shen et al. (2003)), in which the indices for the creep forces are particularly concerned, representing the deviation from equalising forces in lateral direction. Therefore, here the lateral forces and displacements and yaw angles are simultaneously considered as indices to optimise the filter $Q_{2}$ using $H_{\infty}$ techniques.

The simulation results show that the dynamics of curving performance on lateral displacements

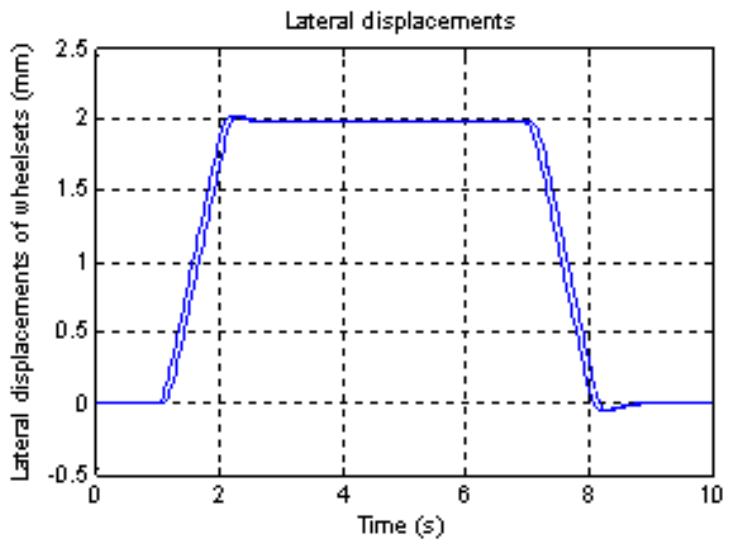

Fig. 6. Lateral displacements with DOBC controller

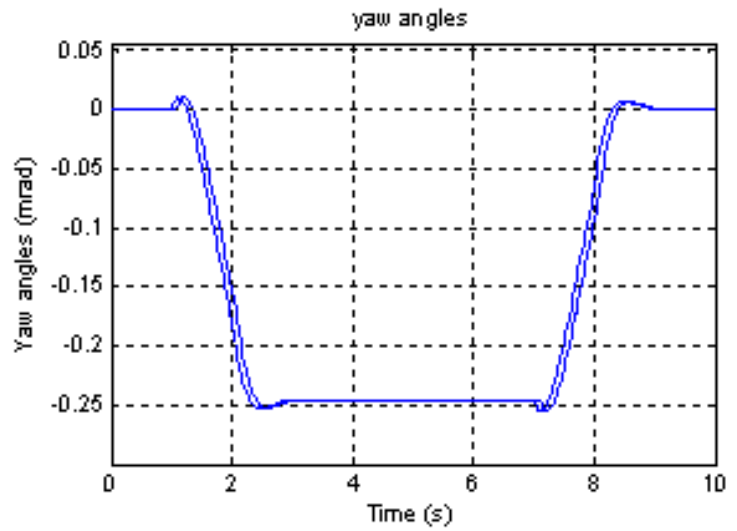

Fig. 7. Yaw angles with DOBC controller

and yaw angles has been improved (see Fig. 6 and Fig. 7), in which the two curves denote the response of leading and trail wheelsets. Although the yaw angles on cured tracks slightly are improved, the lateral forces are significantly reduced (see Fig. 8). So, the curving performance for three indices are improved while the stability on straight tracks does not be interfered. When the railway vehicles run on curved tracks, the filter (controller $Q)$ takes action to improve curving performance due to the output difference between the curved tracks and straight tracks. Since the design of the controller $Q$ is independent of the design of the LQG controller, the added disturbance observer does not have influence on the performance of the LQG controller and when the railway vehicle run on straight tracks, the wheelset stability and performance remains the same. This overcomes the difficulty in compromising the curving performance and the wheelset stability.

\section{CONCLUSIONS}

The DOBC techniques has been extended to MIMO systems using coprime factorisation and then successfully applied to active steering of rail- 


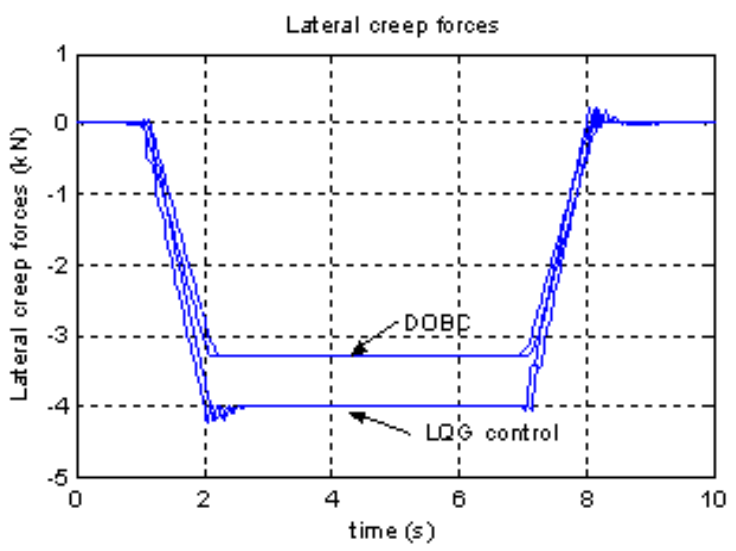

Fig. 8. Comparison of lateral creep forces

way vehicles in this paper. The relationship between the DOBC and the parameterisation of all internally stabilising controllers was established and a new way to parameterisation of all stabilising disturbance observer based controllers was presented. This permits the development of a systematic method to design $Q$ filter, which is one of the main focuses in DOBC, using $H_{\infty}$ control techniques.

Comparing with existing methods for active steering of railway vehicles, simulation demonstrates that the designed DOBC improved the curving performance. The proposed DOBC strategy has the capability of achieving the good curving performance without interference of the vehicle and other performance on straight tracks. This is achieved by first designing an LQG controller for wheelset stability and performance for straight tracks and then a disturbance observer is designed to minimise the influence of the track curves and irregularities on the curving performance. The main benefit for applying DOBC to active steering of railway vehicles is that the tasks of wheelset stability and active steering for curved tracks is completely separated from that of curving performance, which provides an effective and flexible way to deal with active steering problems for railway vehicles.

Acknowledgement: We would like to thank Dr. T. X. Mei and Dr. Ping Li for their clear explanations on the modelling of railway vehicles.

\section{REFERENCES}

Boyd, S., L. Ghaoui, E. Feron, El and V. Balakrishnan (1994). Linear matrix inequalities in systems and control theory. Vol. 15 of Studies in Appl. Math.. SIAM.

Chen, W-H., D. J. Ballance, P. J. Gawthrop and J. O'Reilly (2000). A nonlinear disturbance observer for two-link robotic manipulators. IEEE Trans. on Industrial Electronics 47(4), 932938.
Choi, Y., K. Yang and W. Chung (2003). On the robustness and performance observer for second-order systems. IEEE Trans. on Automatic Control 48(2), 315-320.

Goodall, R. and H. Li (2000). Solid axle and independently rotating wheelsets- a control engineering assessment. Journal of Vehicle System Dynamics 33, 57-67.

MathWorks, Inc. (1997). Lmi control toolbox for matlab.

Mei, T. X. and R. M. Goodall (1999). Optimal control strategies for active steering of railway vehicles. In: IFAC99 World Congress. Vol. F. Beijing. pp. 251-256.

Mei, T. X. and R. M. Goodall (2000a). Lqg and ga solution for active steering of solid axle railway vehicles. Journal of IEE Proceedings Control Theory and Applications 147, 111-117.

Mei, T. X. and R. M. Goodall (2000b). Wheelset control strategies for a 2-axle railway vehicle. Journal of Vehicle System Dynamics 33 Supplement, 653-664.

Mei, T. X. and R. M. Goodall (2003). Recent development in active steering of railway vehicles. Vehicle System Dynamics 39(6), 415-436.

Nett, C. N., C. A. Jacobson and M. J. Balas (1984). A connection between state-space and doubly coprime fractional representations. IEEE Trans. on Automatic Control 29(9), 831832.

Oh, Y., W. K. Chung, Y. Youm and I. H. Suh (1999). Disturbance-observer-based motion control of redundant manipulators using inertially decoupled dynamics. IEEE/ASME Trans. on Mechatronics 4(2), 133-146.

Schrijver, E. and van D. Johannes (2002). Disturbance observers for rigid mechanical systems: equivalence, stability, and design. ASME Journal of Dynamic Systems, Measurement and Control 124(4), 539-548.

Shen, S., T. X. Mei, R. M. Goodall, J. Pearson and G. Himmelstein (2003). Active steering of railway vehicles: A feedforward strategy and assessment of parameter sensitivities. In: European Control Conference. Cambridge, UK.

Umeno, T. and Y. Hori (1991). Robust speed control of dc servomotors using modern two degrees-of-freedom controller design. IEEE Trans on Industrial Electronics.

Vidyasagar, M. (1985). Control system synthesis: a factorisation approach. The MIT Press.

Wickens, A. H. (2003). Fundamentals of rail vehicle dynamics - guidance and stability. Series Advances in Engineering (6). Swets and Zeitlinger.

Zhou, K. and J. C. Doyle (1998). Essentials of Robust Control. Prentice-Hall, Inc. 